\title{
Evolution of supernovae in the winds of massive stars
}

\author{
Vikram V. Dwarkadas \\ Bartol Research Institute, University of Delaware, \\ 217 Sharp Laboratory, Newark, DE 19350, USA
}

\begin{abstract}
Core-collapse supernovae (SNe) arise from massive stars, which lose mass in the form of stellar winds throughout their lifetimes. We study herein the evolution of the SNe as they grow in the circumstellar medium formed by mass loss from the progenitor star.
\end{abstract}

\section{Introduction}

Massive stars give off strong winds, which can substantially modify the medium around the star. Often these winds result in the formation of circumstellar (CS) wind-blown bubbles around the star. At the end of their lives, these stars may die out in a spectacular supernova (SN) explosion. The resulting shockwave will initially evolve within this wind-blown bubble, and thus the evolution of the remnant will be quite different from that in the interstellar medium.

\section{Supernova - circumstellar shell interaction}

The structure of wind-blown bubbles was first elucidated by Weaver et al. (1977). The interaction of SN ejecta with the freely expanding wind from the progenitor star gives rise to a double-shocked structure, consisting of a forward shock driven into the wind and a reverse shock moving into the ejecta. Given the low density interior of the wind-blown bubble, in general most of the bubble mass is contained within a dense circumstellar shell ( $\mathrm{r}_{c s}$ in Figure 1a). Thus the interaction of the ejecta with this shell is crucial to determining the evolution of the remnant. This interaction depends mainly on a single parameter $\Lambda=\frac{M_{\text {shell }}}{M_{\text {ejecta }}}$, the ratio of the shell mass to the ejecta mass.

An exploration of the interaction of SN shock waves with CS bubbles shows (e.g., Dwarkadas 2001, 2002) that, for small values of the parameter $\Lambda \leq 1$, the structure of the density profile is important. Just after the shock-shell interaction has taken place (Figure 1b), the density decreases outwards from the reflected shock to the contact discontinuity. However, as the evolution proceeds, the supernova remnant begins to 'forget' the existence of the shell, and loses memory of the interaction. The density structure changes to reflect this, and begins to increase from the reflected shock to the contact discontinuity (Figure 1c). In this case it takes about 15 doubling times of the radius for the remnant to forget 


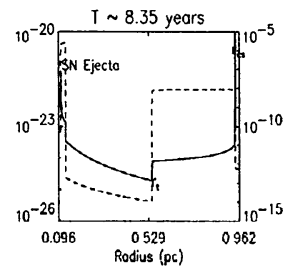

(a)

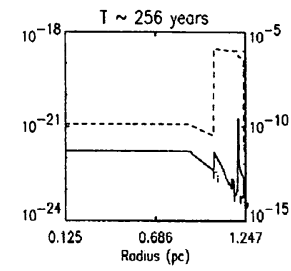

(b)

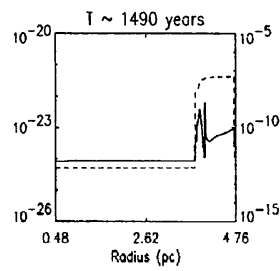

(c)

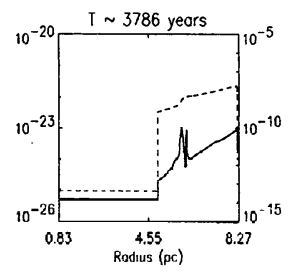

(d)

Figure 1. Density (solid) and pressure (dashed) profiles from a simulation of a SNR evolving in a wind-blown bubble, with $\Lambda=0.14$.

the interaction with the shell (Figure 1d). In another few doubling times, the remnant density profile will resemble that of a SNR evolving directly in the ambient medium. The changing density profile will affect the X-ray and optical emission from the remnant, which depends on the value of the density.

As the value of the parameter $\Lambda$ increases, i.e., the mass of the wind-blown shell increases compared to the ejecta mass, the energy transmitted by the remnant to the shell also increases. Energy transfer to the shell becomes dynamically important, and the remnant evolution is speeded up. The reflected shock, moving rapidly through the ejecta, thermalizes the ejecta in a shorter time than it would take the SN reverse shock to do. If the value of $\Lambda$ is large, the SN outer shock may become radiative, and the kinetic energy is converted to thermal energy. In extreme cases, the remnant may then go directly from the free-expansion stage to the radiative stage, by-passing the classical adiabatic or 'Sedov' stage.

\subsection{A $35 \mathrm{M}_{\odot}$ star}

In order to further explore the evolution of supernovae in a more realistic environment, we have studied the evolution of the medium around a $35 \mathrm{M}_{\odot}$ star, and the resultant interaction of the shock wave with this medium once the star explodes as a SN. The simulations show that density and pressure variations within the wind-blown bubble may give rise to considerable turbulence within the interior. The pressure within the turbulent interior causes the shock wave to become rippled, and may result in an aspherical shock. These computations are further detailed in Dwarkadas (2001; 2002).

Acknowledgments. I would like to thank the IAU for some travel support. My work is supported by NASA grant NAG5-3530, and by a grant from NASA administered by the American Astronomical Society.

\section{References}

Dwarkadas, V.V. 2001, J. Korean Astron. Soc. 34, 243

Dwarkadas, V.V. 2002, in: A.F.J. Moffat \& N. St-Louis (eds.), Interacting Winds from Massive Stars, ASP-CS 260, 141,

Weaver, R.P., McCray, R., Castor, J.I., Shapiro, P.R., Moore, R.L. 1977, ApJ 218, 377 